\title{
METHYL-CpG-BINDING PROTEIN 2 IS LOCALIZED IN THE POSTSYNAPTIC COMPARTMENT: AN IMMUNOCHEMICAL STUDY OF SUBCELLULAR FRACTIONS
}

\begin{abstract}
K. M. ABER, ${ }^{a 1}$ P. NORI, ${ }^{\text {a }}$ S. M. MACDONALD, ${ }^{a}$ G. BIBAT, ${ }^{a, b}$ M. H. JARRAR ${ }^{a, c 1}$ AND W. E. KAUFMANN ${ }^{a, b, c, d, e, f *}$

${ }^{a}$ Kennedy Krieger Institute, 707 North Broadway, Baltimore, MD 21205, USA

${ }^{b}$ Department of Pediatrics, The Johns Hopkins University School of Medicine, 600 North Wolfe Street, Baltimore, MD 21205, USA

'Department of Pathology, The Johns Hopkins University School of Medicine, 600 North Wolfe Street, Baltimore, MD 21205, USA

${ }^{d}$ Department of Neurology, The Johns Hopkins University School of Medicine, 600 North Wolfe Street, Baltimore, MD 21205, USA

${ }^{e}$ Department of Psychiatry and Behavioral Sciences, The Johns Hopkins University School of Medicine, 600 North Wolfe Street, Baltimore, MD 21205, USA

${ }^{f}$ Department of Radiology and Radiological Science, The Johns Hopkins University School of Medicine, 600 North Wolfe Street, Baltimore, MD 21205, USA
\end{abstract}

\begin{abstract}
Methyl-CpG-binding protein 2 is a characteristic member of the methyl-CpG-binding protein family of transcription regulators. In conjunction with $\mathrm{Sin} 3, \mathrm{MeCP} 2$ recruits class I histone deacetylases to methyl-CpG regions to suppress transcription. Rett syndrome, a disorder characterized by mental retardation and autistic features, is associated in a majority of cases with mutations within the coding region of the MeCP2 gene. Considering that defective MeCP2 has mainly been related to Rett syndrome and other neurologic manifestations, we examined methyl-CpG-binding protein 2 cellular and subcellular compartmentalization in normal brain by immunochemical methods. Methyl-CpG-binding protein 2 immunoreactivity is present mainly in neurons; while the few immunostained glia show label confined to nuclei, many neurons also show slight perikaryal staining. Using well-characterized tissue fractions, we found that methyl-CpG-binding protein 2 but not $\operatorname{Sin} 3$ is found in both nuclear and postsynaptic compartments. This novel extranuclear localization is not unique to methyl-CpG-binding protein 2, since it has been previously reported for other transcription regulators such as c-Fos. These findings support the concept that methyl-CpGbinding protein 2 may link synaptic activity and transcriptional regulation in neurons. (C) 2003 IBRO. Published by Elsevier Science Ltd. All rights reserved.
\end{abstract}

${ }^{1}$ Both authors contributed equally to the paper.

${ }^{*}$ Correspondence to: W. E. Kaufmann, Kennedy Krieger Institute, Room 500, 707 North Broadway, Baltimore, MD 21205, USA. Tel: +1-443-923-2789; fax: +1-443-923-2775

E-mail address: wekaufma@jhmi.edu (W. Kaufmann).

Abbreviations: $\mathrm{Ab}$, antibody; CaMK-II, $\mathrm{Ca}(2+)$ /calmodulin-dependent protein kinase II; EDTA, ethylenediaminetetraacetic acid; EGTA, ethylene glycol-bis(2-aminoethyl)-N,N,N',N'-tetraacetic acid; HEPES, 4-(2-hydroxyethyl)piperazine-1-ethanesulfonic acid; IR, immunoreactivity; LP1, lysate pellet 1; LP2, lysate pellet 2; LS1, lysate supernatant 1 ; LS2, lysate supernatant 2 ; MAP-2, microtubule associated protein-2; MeCP2, methyl-CpG-binding protein 2; NeuN, neuronal nuclear antigen; PSD-95, postsynaptic density protein-95; P2, pellet; RS, Rett syndrome.
Key words: MeCP2, Rett syndrome, synaptic fractions, transcription factors.

Rett syndrome (RS) is a developmental neurologic disorder characterized by severe cognitive impairment, autistic behavior, stereotypic movements, and frequently also seizures (Naidu, 1997). The disorder is associated in a majority of cases with mutations of the coding region of the gene for methyl-CpG-binding protein 2 (MeCP2) (Shahbazian and Zoghbi, 2001; Hoffbuhr et al., 2001). More recently, other neurologic syndromes different from RS have been reported as associated with MeCP2 mutations (Imessaoudene et al., 2001; Watson et al., 2001; Dotti et al., 2002). Only limited data are available about the expression of MeCP2 in the CNS. These immunochemical studies describe a predominant neuronal compartmentalization (LaSalle et al., 2001; Shahbazian et al., 2002), and a pattern of expression that parallels neuronal differentiation (Akbarian et al., 2001; Shahbazian et al., 2002). Despite this information, and considering that MeCP2 has wide tissue distribution (LaSalle et al., 2001; Shahbazian et al., 2002), it is still unclear why abnormal MeCP2 expression is mainly associated with neurologic dysfunction. Moreover, recent data show that several transcription factors, with expression in the CNS and other organs, may have a unique neuronal distribution characterized by both nuclear and synaptic localizations (Paratcha et al., 2000; Eberwine et al., 2001). For these reasons, we examined MeCP2 expression in normal human neocortex with special emphasis on its subcellular localization.

\section{EXPERIMENTAL PROCEDURES}

Frozen and formalin-fixed brain samples from two normal female subjects (ages five and 10 years, postmortem interval 36 and $17 \mathrm{~h}$, respectively) were obtained from the Brain and Tissue Bank for Developmental Disorders at the University of Maryland (Baltimore, $M D, ~ U S A)$ and used for all analyses. In addition, fresh frozen (young adult) bovine brain (cerebral cortex) was used as tissue control sample for potential effects of postmortem delay on subcellular fractionation and immunochemical procedures. Fixed posterior frontal tissue was embedded in paraffin and processed for immunocytochemistry as previously described (Kaufmann et al., 2000). Frozen cortical samples from the same frontal region were fractionated to obtain nuclear and synaptic components according to Lewis et al. (1992) and Huttner et al. (1983), respectively. Briefly, nuclear fractions were obtained by homogenization in a buffer containing 20-mM HEPES, 25-mM KCl, 0.5-mM EGTA, 1-mM EDTA, 2-M sucrose, $10 \%$ glycerol, and a cocktail of protease inhibitors, and then layered-centrifugated in a 2.3:1 ratio of tissue homogenate:buffer at $100,000 \times g$. The resulting pellet was 
resuspended in $500 \mu \mathrm{l}$ of denaturing buffer (20-mM HEPES, 5-M urea, 2-M NaCl, $10 \%$ glycerol, $10-\mathrm{mM} \beta$-mercaptoethanol (BME), 1-mM EDTA, 0.5-mM EGTA, pH 7.9), sonicated to fragment DNA, and dialyzed by using a Pierce mini dialysis unit for 2-4 $\mathrm{h}$ (buffer: $50-\mathrm{mM} \mathrm{NaCl}, 20-\mathrm{mM}$ HEPES, 1-mM phenylmethanesulfonyl fluoride (PMSF), 1-mM EGTA, pH 7.9) according to the manufacturer's instructions. Synaptic fractions were obtained at $4{ }^{\circ} \mathrm{C}$ by homogenizing frozen cerebral cortex in a sucrose-HEPES buffer (0.32-M sucrose, 4-mM HEPES- $\mathrm{NaOH}, \mathrm{pH} 7.3$, and a cocktail of protease inhibitors) at $800 \times g$ for $10 \mathrm{~min}$. The resulting supernatant was centrifuged at $9200 \times g$ for $15 \mathrm{~min}$, yielding a pellet (P2) that was resuspended in the same buffer and centrifuged at $10,200 \times g$ for $15 \mathrm{~min}$. The resulting pellet (P2') was resuspended in sucrose-HEPES buffer and lysed by hyposmotic shock in order to disrupt membranes, followed by mixing with one volume of 7.5-mM HEPES- $\mathrm{NaOH}(\mathrm{pH} 7.4$ ) and cooling. This processed P2' pellet corresponded to the lysed synaptosomal fraction, which was centrifuged at $25,000 \times g$ for 20 min to yield both lysate pellet 1 (LP1), a membrane-enriched combined pre- and postsynaptic fraction, and its soluble counterpart, lysate supernatant 1 (LS1). Centrifugation of LS1 at $165,000 \times g$ for $2 \mathrm{~h}$ resulted in lysate supernatant 2 (LS2) and lysate pellet 2 (LP2), both predominantly presynaptic fractions. Due to the high lipid content of the membrane-enriched synaptic LP1 and LP2 fractions, we performed trichloroacetic acid precipitation on the samples to allow a better protein resolution (Crane et al., 1994). Nuclear and synaptic fractions LP1, LP2 and LS2 were separated by standard, using $8 \%-$ $10 \%$ precast gel from ISC BioExpress (Kaysville, UT, USA), and immunoblotted as reported (Kaufmann et al., 1996, 1997; Sun et al., 2001). Three MeCP2 antibodies (Ab) were used: a full-length affinity-purified rabbit polyclonal $A b$ against the methyl-binding domain of Xenopus MeCP2 (Yusufzai and Wolffe, 2000), kindly provided by Dr. Peter Jones (NICHD, Bethesda, MD, USA); a rabbit polyclonal $\mathrm{Ab}$ that targets a specific $\mathrm{C}$-terminal sequence of the mouse protein (residues 465-478) from Upstate (Lake Placid, NY, USA; Lewis et al., 1992; LaSalle et al., 2001); and a mouse monoclonal $\mathrm{Ab}$ directed against an amino-terminal epitope (residues 1-138) of recombinant human protein, a courtesy from Dr. Paul Wade (Emory University, Atlanta, GA, USA). Antibodies for tissue-fraction characterization included postsynaptic density protein-95 (PSD-95) and neuronal nuclear antigen (NeuN) from Chemicon (Temecula, CA, USA); SMI-52, against microtubuleassociated protein-2 (MAP-2) from Sternberger (Lutherville, MD, USA); synaptophysin from Biogenex (San Ramon, CA, USA); the nuclear marker DNA polymerase- $\beta$ from Labvisions Corporation (Fremont, CA, USA); and the nuclear and postsynaptic protein $\mathrm{Ca}$ $(2+) /$ calmodulin-dependent protein kinase II (CaMK-II) from Sigma (Saint Louis, MO, USA). Antibodies directed at transcriptional regulators included Egr-1 and Sin $3 \mathrm{~A}$ from Santa Cruz Biotechnology (Santa Cruz, CA); c-Fos specific for an amino-terminal epitope (residues 3-16) of the human protein from Sigma (Saint Louis, MO, USA); and Abs against c-Fos and c-Jun, both kindly provided by the late Dr. Daniel Nathans (The Johns Hopkins University, Baltimore, MD, USA).

\section{RESULTS}

Immunocytochemical characterization of MeCP2 cellular distribution in the frontal cortex demonstrated a predominant neuronal distribution, with a few glial immunoreactive nuclei particularly in the white matter. Neurons showed mainly a mottled nuclear staining, which was variable in intensity from cell to cell. In addition, neurons with intense MeCP2 nuclear immunoreactivity (IR), mainly of the pyramidal type, also showed weak cytoplasmic staining that was confined to the perikaryal region and proximal dendrites (Fig. 1). MeCP2 neuronal cytoplasmic staining was

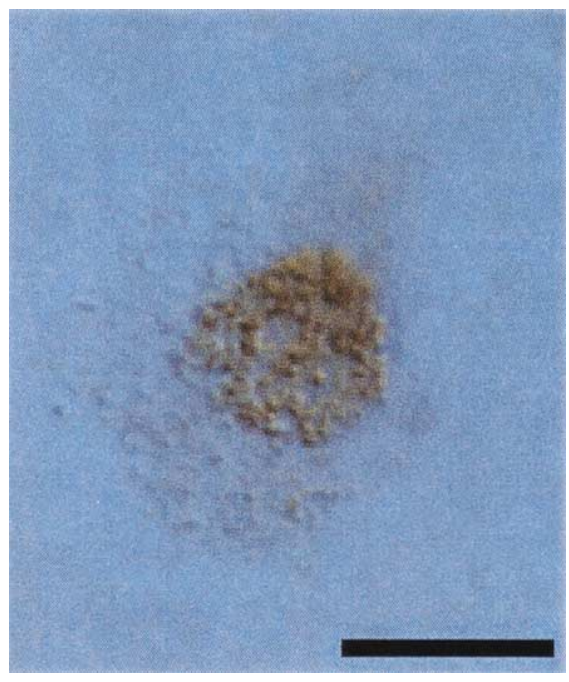

Fig. 1. Methyl-CpG-binding protein 2 (MeCP2) immunocytochemical pattern in human frontal cortex. Layer VI pyramidal neuron showing mottled nuclear MeCP2 immunoreactivity with a similar, but less intense, staining in the perikaryal region. This cytoplasmic pattern was only found in neurons with intense nuclear immunolabeling. Scale bar $=10 \mu \mathrm{m}$

further characterized by tissue fractionation using standard protocols (Lewis et al., 1992; Huttner et al., 1983). Considering that the protocol used for obtaining synaptic fractions had mainly described them from an ultrastructural but not from an immunochemical perspective (Huttner et al., 1983), we first examined the profile of characteristic preand postsynaptic markers (Weidenmann and Franke, 1985; Kennedy, 1998; Kaufmann et al., 2000). The "enriched synaptosomal fraction," also termed LP1 fraction (Huttner et al., 1983), was characterized by IR for PSD-95, MAP-2, and synaptophysin, while the high-speed pellet referred to as "synaptic microsomal fraction" (LP2) (Huttner et al., 1983), and its high-speed supernatant counterpart (LS2; not shown) showed only IR for the presynaptic marker synaptophysin (Fig. 2A). DNA polymerase $\beta$, our marker for nuclear fractions, was not detected in either LP1 or LP2 (Fig. 2A). Immunoblotting of nuclear and synaptic fractions with three different MeCP2 Ab targeting distinct regions of the protein demonstrated IR in nuclear and LP1 fractions, but not in either LP2 or LS2 (Fig. 2B).

In order to determine whether synaptic localization of MeCP2 was a feature of other transcriptional regulators in human neocortex, as demonstrated in rodents (Paratcha et al., 2000; Eberwine et al., 2001) and other vertebrates (Cobellis et al., 1999), we examined the subcellular distribution of c-Fos, c-Jun, Egr1, NeuN, and Sin3. The latter is a protein that, by interacting with MeCP2, recruits class I histone deacetylases to methyl-CpG regions (Nan et al., 1998; Jones et al., 1998; Kokura et al., 2001). As shown in Fig. 3, while Egr1, NeuN, and Sin3 were only found in nuclear fractions, immunoreactivity for C-Fos and C-Jun was also detected in the LP1 fraction (Fig. 3A). No immunoreactivity for any transcriptional regulator was found in either LP2 or LS2. Additional support for the validity of the MeCP2 findings was provided by the distribution of CaMK-II. As recently reported 
A

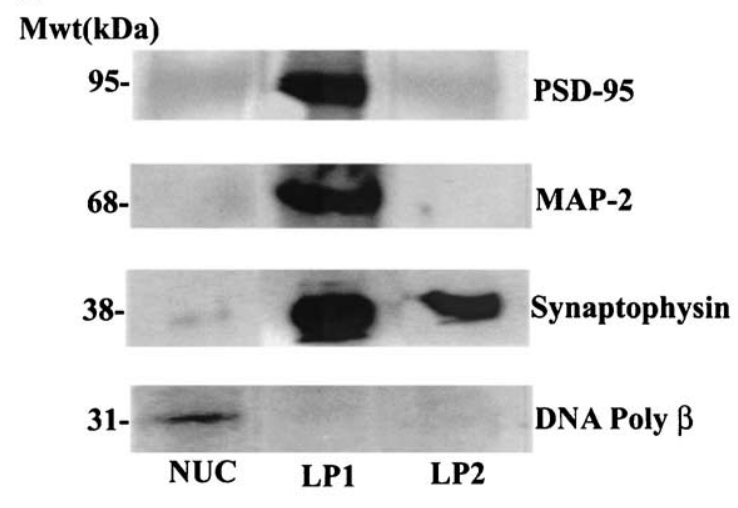

B

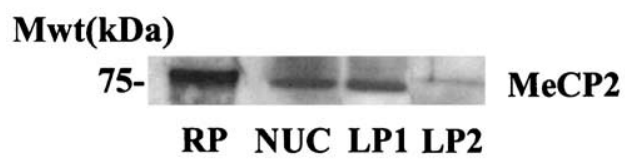

Fig. 2. Methyl-CpG-binding protein 2 (MeCP2) immunoreactivity in human frontal cortical tissue fractions. (A) Immunochemical profile of nuclear (NUC) and synaptic (lysate supernatant 1 [LP1], lysate supernatant 2 [LP2]) fractions. Postsynaptic markers density protein-95 and microtubule associated protein-2 were only present in LP1 fractions, while the presynaptic protein synaptophysin was detected in both LP1 and LP2. Minimal immunoreactivity for synaptophysin is present in NUC, which is intensely positive for DNA polymerase $\beta$. (B) MeCP2 immunoreactivity is found in both NUC and LP1 fractions, and minimally in LP2. RP corresponds to human recombinant MeCP2. The figure depicts immunoreactivity for a rabbit polyclonal $A b$ that targets residues 465-478 of mouse MeCP2 (Upstate Biotechnology). Identical results were obtained when the two other MeCP2 Abs mentioned in the Experimental Procedures section were used. Molecular weight (Mwt) sizes in kilodaltons $(\mathrm{kDa})$ are shown on the left.

(Yamauchi and Yoshimura, 1998; Laabich et al., 2000), this major postsynaptic protein was found in both LP1 and nuclear fractions, but not in the predominantly presynaptic fractions (Fig. 3B). All the aforementioned results were found in both bovine and human tissue fractions.

\section{DISCUSSION}

It is well established that MeCP2 plays an important role in transcriptional repression (Nan et al., 1998), which is related to its association with heterochromatin in the nuclear compartment (Lewis et al., 1992). Recently reported mutations in the coding region of MeCP2 associated with RS (Lee et al., 2001) lead to protein truncations that alter this nuclear compartmentalization, resulting in a more diffuse pattern of MeCP2 nuclear IR (Kudo et al., 2002). Furthermore, there is increasing evidence indicating that in neurons, transcription factors may have an additional synaptic localization (Paratcha et al., 2000; Eberwine et al., 2001). Our examination of the cellular and subcellular distribution of MeCP2 in human neocortex confirms previous reports of a predominantly neuronal distribution (Akbarian et al., 2001; LaSalle et al., 2001; Shahbazian et al., 2002). We found neurons with different levels of MeCP2 nuclear immunostaining, as previously communicated (LaSalle et al.,

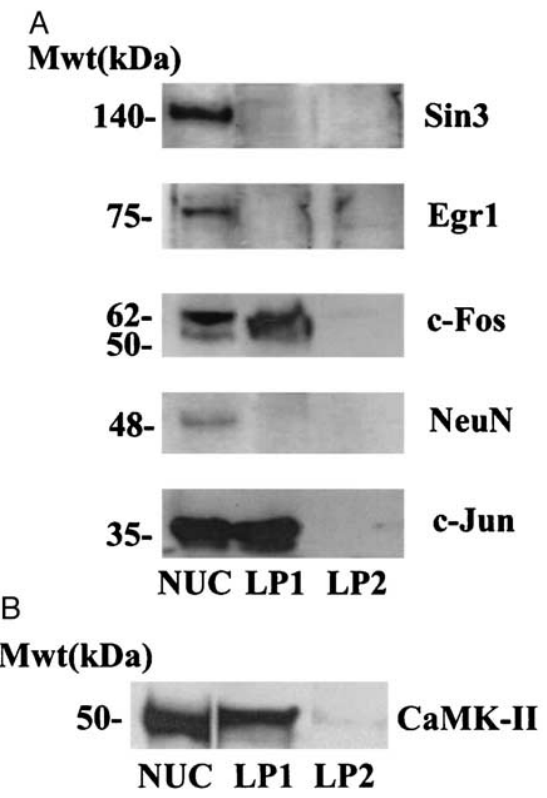

Fig. 3. Immunoblotting profile of selected nuclear proteins. (A) Among major neuronal transcriptional regulators, only c-Fos and c-Jun are found in the lysate supernatant 1 (LP1) synaptic fraction. No marker was found in the predominantly presynaptic lysate supernatant 2 fraction. (B) As previously reported (Laabich et al., 2000), CaMK-II immunoreactivity is detected in both NUC and pre- and postsynaptic LP1 fraction. Molecular weight (Mwt) sizes in kilodaltons (kDa) are shown on the left.

2001). However, we also demonstrated that, in at least a subset of neurons, there is cytoplasmic MeCP2 IR. This rather perikaryal distribution was further corroborated by tissue fractionation assays, which showed MeCP2 IR in a mixed pre+postsynaptic fraction but not in a presynaptic fraction. As expected from recent studies (Paratcha et al., 2000; Eberwine et al., 2001), MeCP2 postsynaptic localization is shared by other transcriptional regulators. c-Fos and C-Jun also appear to be postsynaptic, despite a report indicating that C-Fos is presynaptic and C-Jun is not in synaptic regions (Paratcha et al., 2000).

Although we did not detect MeCP2 IR in distal dendrites, our data suggest that MeCP2 may be regulated in a manner similar to the cyclic AMP responsive element binding protein. This transcription factor is synthesized locally at the dendritic level, on the basis of transcripts translocated from the nucleus and in response to synaptic activity (Eberwine et al., 2001). The circumscription of MeCP2's interactor Sin3 to nuclear fractions suggests that MeCP2 may be the only element of this transcriptional repression complex to be under direct synaptic influence. A predominant perikaryal MeCP2 distribution indicates a potential role in transcriptional regulation secondary to inhibitory synaptic activity (Grantyn et al., 1995). These findings regarding MeCP2 are not unique to transcriptional regulators, but also involve other signaling molecules in neurons. Recent data on CaMK-II demonstrate that, in addition to its critical postsynaptic function, this kinase is also present in neuronal nuclei (Yamauchi and Yoshimura, 1998; Laabich et al., 2000), as we also showed in human frontal cortex. The wide brain distribution of MeCP2 and its 
developmental profile (Shahbazian et al., 2002), which parallels neuronal differentiation, suggest that this protein is critical for neuronal development and function. An additional synaptic localization will only further emphasize this role. We postulate that the preferential neurologic phenotype (i.e. RS, nonspecific mental retardation) associated with MeCP2 mutations may be explained, at least in part, by a unique role for this protein in neuronal function. Furthermore, abnormal neuronal compartmentalization of truncated MeCP2 could result in disrupted synaptic function leading to severe neurologic manifestations, as those typically seen in RS. Additional studies are required to explore these hypotheses.

Acknowledgements-This work was supported by NIH grants HD24448 and HD24061. The authors thank Peter Jones for providing MeCP2 antibodies and recombinant protein, the late Daniel Nathans for providing the c-Fos and c-Jun antibodies, Paul Watkins for assistance in tissue sample preparation, Mollie Lange for advice on immunocytochemistry, and Sakkubai Naidu for continuous support of the project.

\section{REFERENCES}

Akbarian S, Chen RZ, Gribnau J, Rasmussen TP, Fong H, Jaenisch R, Jones EG (2001) Expression pattern of the Rett syndrome gene MeCP2 in primate prefrontal cortex. Neurobiol Dis 8:784-791.

Cobellis G, Vallarino M, Meccariello R, Pierantoni R, Masini MA, Mathieu M, Pernas-Alonso R, Chieffi P, Fasano S (1999) Fos localization in cytosolic and nuclear compartments in neurones of the frog, Rana esculenta, brain: an analysis carried out in parallel with GnRH molecular forms. J Neuroendocrinol 11:725-735.

Crane DI, Kalish JE, Gould SJ (1994) The Pishcia pastoris PAS4 gene encodes a ubiquitin-conjugating enzyme required for peroxisome assembly. J Biol Chem 269:21835-21844.

Dotti MT, Orrico A, De Stefano N, Battisti C, Sicurelli F, Severi S, Lam CW, Galli L, Sorrentino V, Federico A (2002) A Rett syndrome Mecp2 mutation that causes mental retardation in men. Neurology 58:226-230.

Eberwine J, Miyashiro K, Kacharmina JK, Job C (2001) Local translation of classes of mRNAs that are targeted to neuronal dentrites. Proc Natl Acad Sci USA 98:7080-7085.

Grantyn R, Kraszewski K, Melnick I, Taschenberger H, Warton SS (1995) In vitro development of vertebrate central synapses. Perspect Dev Neurobiol 2:387-397.

Hoffbuhr K, Devaney JM, LaFleur B, Sirianni N, Scacheri C, Giron J, Schuette J, Innis J, Marino M, Philippart M, Narayanan V, Umansky R, Kronn D, Hoffman EP, Naidu S (2001) MeCP2 mutations in children with and without the phenotype of Rett syndrome. Neurology 56:1486-1495.

Huttner WB, Scheibler W, Greengard P, De Camilli P (1983) Synapsin I (protein I), a nerve terminal-specific phosphoprotein. III. Its association with synaptic vesicle preparation. J Cell Biol 96:1374-1388.

Imessaoudene B, Bonnefont JP, Royer G, Cormier-Daire D, Lyonnet S, Lyon G, Munnich A, Amiel J (2001) MECP2 mutation in non-fatal, non-progressive encephalopathy in a male. J Med Genet 38:171-174.

Jones PL, Veenstra GJ, Wade PA, Vermaak D, Kass SU, Landsberger N, Strouboulis J, Wolffe AP (1998) Methylated DNA and MeCP2 recruit histone deacetylases to repress transcription. Nat Genet 19:187-191.

Kaufmann WE, Worley PF, Pegg J, Bremer M, Isakson P (1996) Cox-2, a synaptically induced enzyme, is expressed by excitatory neurons at postsynaptic sites in rat cerebral cortex. Proc Natl Acad Sci USA 93:2317-2321.

Kaufmann WE, Taylor CV, Lishaa NA (1997) Immunoblotting patterns of cytoskeletal dendritic protein expression in human neocortex. Mol Chem Neuropathol 31:235-244.

Kaufmann WE, MacDonald SM, Altamura CR (2000) Dendritic cytoskeletal expression in mental retardation: an immunohistochemical study of neocortex in Rett syndrome. Cereb Cortex 10:9921004.

Kennedy MB (1998) Signal transduction molecules at the glutamatergic postsynaptic membrane. Brain Res Rev 26:243-257.

Kokura K, Kaul SC, Wadhwa R, Nomura T, Khan MM, Shinagawa T, Yasukawa T, Colmenares C, Ishii S (2001) The Ski protein is required for MeCP2-mediated transcriptional repression. J Biol Chem 276:34115-34121.

Kudo S, Nomura Y, Segawa M, Fujita N, Nakao M, Hammer S, Schanen C, Teri I, Tamura M (2002) Functional characterization of Mecp2 mutations found in male patients with $X$ linked mental retardation. J Med Genet 39:132-136.

Laabich A, Li G, Cooper NG (2000) Calcium/calmodulin-dependent protein kinase II containing a nuclear localization signal is altered in retinal neurons exposed to N-methyl-D-aspartate. Mol Brain Res 76:253-265.

LaSalle JM, Goldstine J, Balmer D, Greco CM (2001) Quantitative localization of heterogeneous methyl-CpG-binding protein 2 (MeCP2) expression phenotypes in normal and Rett syndrome brain by laser scanning cytometry. Hum Mol Genet 10:1729-1734.

Lee SSJ, Wan M, Franke U (2001) Spectrum of Mecp2 mutations in Rett syndrome. Brain Dev 23:138-148.

Lewis JD, Meehan RR, Henzel WJ, Maurer-Fogy I, Jeppesen P, Klein F, Bird A (1992) Purification, sequence, and cellular localization of a novel chromosomal protein that binds to methylated DNA. Cell 105:487-497.

Naidu S (1997) Rett syndrome: a disorder affecting early brain growth. Ann Neurol 42:3-10.

Nan X, Ng HH, Johnson CA, Laherty CD, Turner BM, Eisenman RN, Bird A (1998) Transcriptional repression by the methyl-CpG-binding protein MeCP2 involves a histone deacetylase complex. Nature 393:386-389.

Paratcha G, Stein ML, Szapiro G, Lopez M, Bevilaqua L, Cammarota M, Iraldi AP, Izquierdo I, Medina JH (2000) Experience-dependent decrease in synaptically localized Fra-1. Mol Brain Res 70:120-130.

Shahbazian MD, Antalffy B, Armstrong DL, Zoghbi HY (2002) Insight into Rett syndrome: MeCP2 levels display tissue- and cell-specific differences and correlate with neuronal maturation. Hum Mol Genet 11:115-124.

Shahbazian MD, Zoghbi HY (2001) Molecular genetics of Rett syndrome and clinical spectrum of Mecp2 mutations. Curr Opin Neurol 14:171-176.

Sun HT, Cohen S, Kaufmann WE (2001) Annexin-1 is abnormally expressed in Fragile $\mathrm{X}$ syndrome: two-dimensional electrophoresis study in lymphocytes. Am J Med Genet 103:81-90.

Watson P, Black G, Ramsden S, Barrow M, Super M, Kerr B, ClaytonSmith J (2001) Angelman syndrome phenotype associated with mutations in MECP2, a gene encoding a methyl CpG binding protein. J Med Genet 38:224-228.

Weidenmann B, Franke WW (1985) Identification and localization of synaptophysin, an integral membrane glycoprotein of $M_{r} 38,000$ characteristic of presynaptic vesicles. Cell 41:1017-1028.

Yamauchi T, Yoshimura Y (1998) Phosphorylation-dependent reversible translocation of $\mathrm{Ca} 2+/$ calmodulin-dependent protein kinase II to the postsynaptic densities. Life Sci 62:1617-1621.

Yusufzai TM, Wolffe AP (2000) Functional consequences of Rett syndrome mutations on human Mecp2. Nucleic Acids Res 28:4172-4179. 